\title{
Small Intestinal Bacterial Overgrowth in Patients with Refractory Functional Gastrointestinal Disorders
}

\author{
Shino Shimura, Norihisa Ishimura, ${ }^{*}$ Hironobu Mikami, Eiko Okimoto, Goichi Uno, Yuji Tamagawa, Masahito Aimi, Naoki Oshima, \\ Shuichi Sato, Shunji Ishihara, and Yoshikazu Kinoshita \\ Department of Gastroenterology and Hepatology, Shimane University School of Medicine, Izumo, Shimane, Japan
}

\section{Background/Aims}

Small intestinal bacterial overgrowth (SIBO) is considered to be involved in the pathogenesis of functional gastrointestinal disorders (FGID). However, the prevalence and clinical conditions of SIBO in patients with FGID remain to be fully elucidated. Here, we examined the frequency of SIBO in patients with refractory FGID.

\section{Methods}

We prospectively enrolled patients with refractory FGID based on Rome III criteria. A glucose hydrogen breath test (GHBT) was performed using a gas analyzer after an overnight fast, with breath hydrogen concentration measured at baseline and every 15 minutes after administration of glucose for a total of 3 hours. A peak hydrogen value $\geq 10 \mathrm{ppm}$ above the basal value between 60 and 120 minutes after administration of glucose was diagnosed as SIBO.

\section{Results}

A total of 38 FGID patients, including 11 with functional dyspepsia (FD), 10 with irritable bowel syndrome (IBS), and 17 with overlapping with FD and IBS, were enrolled. Of those, 2 (5.3\%) were diagnosed with SIBO (one patient diagnosed with FD; the other with overlapping FD and IBS). Their symptoms were clearly improved and breath hydrogen levels decreased to normal following levofloxacin administration for 7 days.

\section{Conclusions}

Two patients initially diagnosed with FD and IBS were also diagnosed with SIBO as assessed by GHBT. Although the frequency of SIBO is low among patients with FGID, it may be important to be aware of SIBO as differential diagnosis when examining patients with refractory gastrointestinal symptoms, especially bloating, as a part of routine clinical care.

(J Neurogastroenterol Motil 2016;22:60-68)

Key Words

Adult; Bacterial overgrowth syndrome; Blind loop syndrome; Breath tests; Dyspepsia

Received: July 2, 2015 Revised: October 17, 2015 Accepted: November 2, 2015

(a) This is an Open Access article distributed under the terms of the Creative Commons Attribution Non-Commercial License (http://creativecommons. org/licenses/by-nc/4.0) which permits unrestricted non-commercial use, distribution, and reproduction in any medium, provided the original work is properly cited.

${ }^{*}$ Correspondence: Norihisa Ishimura, MD, PhD Department of Gastroenterology and Hepatology, Shimane University School of Medicine, 89-1 Enya-cho, Izumo, Shimane 6938501, Japan

Tel: +81-853-20-2190, Fax: +81-853-20-2187, E-mail: ishimura@med.shimane-u.ac.jp 


\section{Introduction}

Functional gastrointestinal disorders (FGID) are among the most common disorders of the gastrointestinal tract reported in visits to health care providers and have a large impact on health care costs. The prevalence of FGID among general populations worldwide is approximately $20-30 \% .^{1-3}$ Although the pathophysiological mechanisms underlying these disorders are not completely understood, dysmotility, visceral hypersensitivity, abnormal brain-gut interaction, microbiome differences, psychiatric co-morbidity, and immune activation have been suggested to play important roles. ${ }^{4-8}$ Recently, a considerable number of studies have suggested that gut flora plays an important role in occurrence of symptoms and possibly the pathogenesis of FGID. ${ }^{9-11}$ Bacterial flora in the proximal small intestine generally consists of aerobe and facultative anaerobic bacteria at $\leq 10^{4}$ colony forming units $(\mathrm{CFU}) / \mathrm{mL}$ in healthy subjects, while an abnormally high microbial population level in the small intestine is known as small intestinal bacterial overgrowth (SIBO). ${ }^{12}$ Recent studies have suggested that SIBO may have a role in the pathogenesis of FGID.

Conventionally, a diagnosis of SIBO is based on jejunal aspirate culture findings. However, the technique for sample collection is invasive and because of limited access by the instrumentation generally utilized, patients with isolated distal SIBO remain undiagnosed. On the other hand, noninvasive hydrogen breath tests using glucose or lactulose have been widely used as a diagnostic tool to establish SIBO, though their accuracy remains in dispute. ${ }^{13}$ Greater diagnostic accuracy has been demonstrated for a glucose hydrogen breath test (GHBT) with fewer false-positive results as compared to a lactulose hydrogen breath test (LHBT). ${ }^{14,15}$

SIBO is characterized by various gastrointestinal symptoms such as abdominal pain, bloating, distention, and diarrhea, which are found in patients with FGID, and may be associated with excessive gas in the small intestine due to the presence of a large number of hydrogen-producing bacteria. Indeed, it has been demonstrated that patients experiencing functional dyspepsia (FD) or irritable bowel syndrome (IBS) have a higher prevalence of abnormal microbial fermentation in the small intestine as compared with healthy controls, ${ }^{16-18}$ with the prevalence reported to be $56.5 \%$ in patients with $\mathrm{FD}^{16}$ and ranging from $2-84 \%$ in those with IBS. ${ }^{19-21}$ These results support the hypothesis that SIBO may play a role in FGID. Conventional treatment for FGID, such as prokinetics, proton pump inhibitors (PPIs), and antidepressants, may not be effective for symptoms occurring in patients with SIBO. Therefore, the frequency of SIBO in patients with refractory or intractable FGID is likely greater as compared with that in those with well-controlled symptoms.

In the present study, we examined the frequency of SIBO in patients with refractory FGID using GHBT. From our findings, we also discuss the utility of and problems with hydrogen breath testing.

\section{Materials and Methods}

\section{Enrolled Patients}

This study was conducted at Shimane University Hospital and a related facility (Oki Hospital) between July 2013 and January 2015. We prospectively enrolled patients with refractory FGID, defined as the condition which was failed initial treatment, including prokinetics, acid inhibitory drugs, antidepressants for at least 4 weeks. Enrolled patients were confirmed to have no organic cause of their abdominal symptoms when assessed by blood and fecal test, endoscopic examination, and radiology test (ie, computed tomography) findings, and diagnosed with FGID according to the Rome III criteria. ${ }^{1,22} \mathrm{FD}$ patients were further subtyped into 2 group; those with postprandial distress syndrome (PDS), and those with epigastric pain syndrome (EPS). Likewise, IBS patients were further subtyped, on the basis of bowel habits, into case with diarrhea, constipation, and mixed bowel pattern. Those less than 18 years of age, with a history of gastrointestinal surgery (except appendectomy), diabetes mellitus, connective tissue disease, liver disease, renal disease, neurologic disorders, or who had taken antibiotics or probiotics within 4 weeks preceding the test were excluded. A group of 35 healthy volunteers (15 men, 20 women; mean age 23.7 years, range 20-32 years) were also enrolled as controls. All of the control subjects had neither gastrointestinal symptoms nor items defined as exclusion criteria.

\section{Symptom Questionnaire}

Abdominal symptoms in enrolled patients were assessed using the Izumo scale prior to the hydrogen breath test. The Izumo scale was developed and validated by our group, ${ }^{23,24}$ and is widely utilized in Japan ${ }^{25,26}$ as a self-administered questionnaire regarding the effects of abdominal symptoms on quality of life over the past 7 days (Table 1). The survey includes 15 items in 5 domains: reflux (questions 1-3), pain (questions 4-6), fullness (questions 7-9), constipation (question 10-12), and diarrhea (questions 13-15), with 3 items in each domain. Each question is rated on a 6-point Likert 
Table 1. The Izumo Scale Questionnaire Respondents Enter the Severity Scores After Reflecting on Their Symptoms over the Previous Week

\begin{tabular}{|c|c|c|c|c|c|c|}
\hline Question & $\begin{array}{c}\text { Not } \\
\text { bothered }\end{array}$ & $\begin{array}{c}\text { Not so } \\
\text { bothered }\end{array}$ & $\begin{array}{l}\text { Slightly } \\
\text { bothered }\end{array}$ & Bothered & $\begin{array}{l}\text { Strongly } \\
\text { bothered }\end{array}$ & $\begin{array}{c}\text { Intolerably } \\
\text { bothered }\end{array}$ \\
\hline 1. Are you bothered by acid reflux? & 0 & 1 & 2 & 3 & 4 & 5 \\
\hline 2. Are you bothered by heartburn centered in the anterior chest? & 0 & 1 & 2 & 3 & 4 & 5 \\
\hline 3. Are you bothered by throat discomfort? & 0 & 1 & 2 & 3 & 4 & 5 \\
\hline 4. Are you bothered by epigastric pain? & 0 & 1 & 2 & 3 & 4 & 5 \\
\hline 5. Are you bothered by hunger epigastric pain? & 0 & 1 & 2 & 3 & 4 & 5 \\
\hline 6. Are you bothered by epigastric burning sensation? & 0 & 1 & 2 & 3 & 4 & 5 \\
\hline 7. Are you bothered by early satiation? & 0 & 1 & 2 & 3 & 4 & 5 \\
\hline 8. Are you bothered by post-prandial long-lasting epigastric fullness or nausea? & 0 & 1 & 2 & 3 & 4 & 5 \\
\hline 9. Are you bothered by epigastric bloating? & 0 & 1 & 2 & 3 & 4 & 5 \\
\hline 10. Are you bothered by a feeling of incomplete defecation? & 0 & 1 & 2 & 3 & 4 & 5 \\
\hline 11. Are you bothered by constipation or hard stools? & 0 & 1 & 2 & 3 & 4 & 5 \\
\hline 12. Are you bothered by stress-related constipation? & 0 & 1 & 2 & 3 & 4 & 5 \\
\hline 13. Are you bothered by fecal urgency? & 0 & 1 & 2 & 3 & 4 & 5 \\
\hline 14. Are you bothered by diarrhea or soft stools? & 0 & 1 & 2 & 3 & 4 & 5 \\
\hline 15. Are you bothered by stress-related diarrhea? & 0 & 1 & 2 & 3 & 4 & 5 \\
\hline
\end{tabular}

scale from 0 to 5 , with higher values indicating more severe symptoms. The total score ranges from 0 to 75 , with a score of 0 to 15 for each category. Most bothersome symptom, which was defined as troublesome gastrointestinal symptoms with moderate to severe interfering with daily activity in each patient was also asked (duplicate counted if several symptoms were bothered equally). Abnormal bowel movements were defined as bowel frequency with more than 3 times per day or less than 3 times per week. Stool consistency assessed by Bristol stool form scale (type 1, 2, 6, or 7) was also considered as diagnosing abnormal bowel movements. ${ }^{27}$

\section{Glucose Hydrogen Breath Test}

The presence of SIBO was determined using a GHBT, which was performed after an overnight fast along with a carbohydrate restricted diet the previous 24 hours. Drugs for the treatment of FGID were withdrawn 24 hours before the test. In addition, cigarette smoking and exercise were prohibited 2 hours before and during the test. Before starting the GHBT, patients brushed their teeth and washed their mouth with Listerine mouthwash (Johnson and Johnson, McNeil-PPC, USA) to avoid false positive high basal levels from substrate fermentation by oral bacteria. Breath hydrogen tests were performed using a portable hand-held breath hydrogen meter $\left(\right.$ Gastro $^{+}$Gastrolyzer, Bedfront Scientific, Kent, UK). This hydrogen meter is widely used and validated for the assessment of SIBO. ${ }^{28-30}$ Hydrogen gas concentration was expressed in parts per million (ppm) at that instant. After providing a baseline breath sample, the patient ingested $50 \mathrm{~g}$ of glucose in $300 \mathrm{~mL}$ of water. ${ }^{15}$
After gargling, breath samples were taken every 15 minutes for 3 hours.

\section{Diagnosis of Small Intestinal Bacterial Overgrowth}

GHBT patterns were classified as normal, SIBO, and indeterminate (Fig. 1). The normal pattern was defined as a basal value $<10$ ppm with subtracted peak value above the basal $<10$ ppm. SIBO was diagnosed when the basal value was $<10 \mathrm{ppm}$ and the subtracted peak value above basal was $\geq 10$ ppm at 60 120 minutes after glucose loading. ${ }^{15,31}$ The indeterminate pattern was defined as neither the normal nor SIBO pattern, such as basal value $>10 \mathrm{ppm}$ and the subtracted peak value above the basal at $\geq 10$ ppm within 60 or $>120$ minutes after glucose loading. This pattern includes insufficient preparation for breath testing, such as poor adherence to restricted diet, fasting time, cigarette smoking, and exercise, and carbohydrate malabsorption. ${ }^{15}$

\section{Treatment with Antibiotics}

Patients positive for SIBO were treated with levofloxacin at $500 \mathrm{mg}$ for 7 days. Although rifaximin is used worldwide for treatment of SIBO, ${ }^{32,33}$ it has not been adopted in Japan, thus we used levofloxacin, which is commonly used for intestinal bacterial infection with a broad spectrum of activity against Gram-negative and Gram-positive bacteria. One month after the end of treatment, patients were retested for the presence of SIBO by GHBT. Response to levofloxacin was considered positive if GHBT showed a normal pattern and symptoms were improved from the baseline. In addi- 


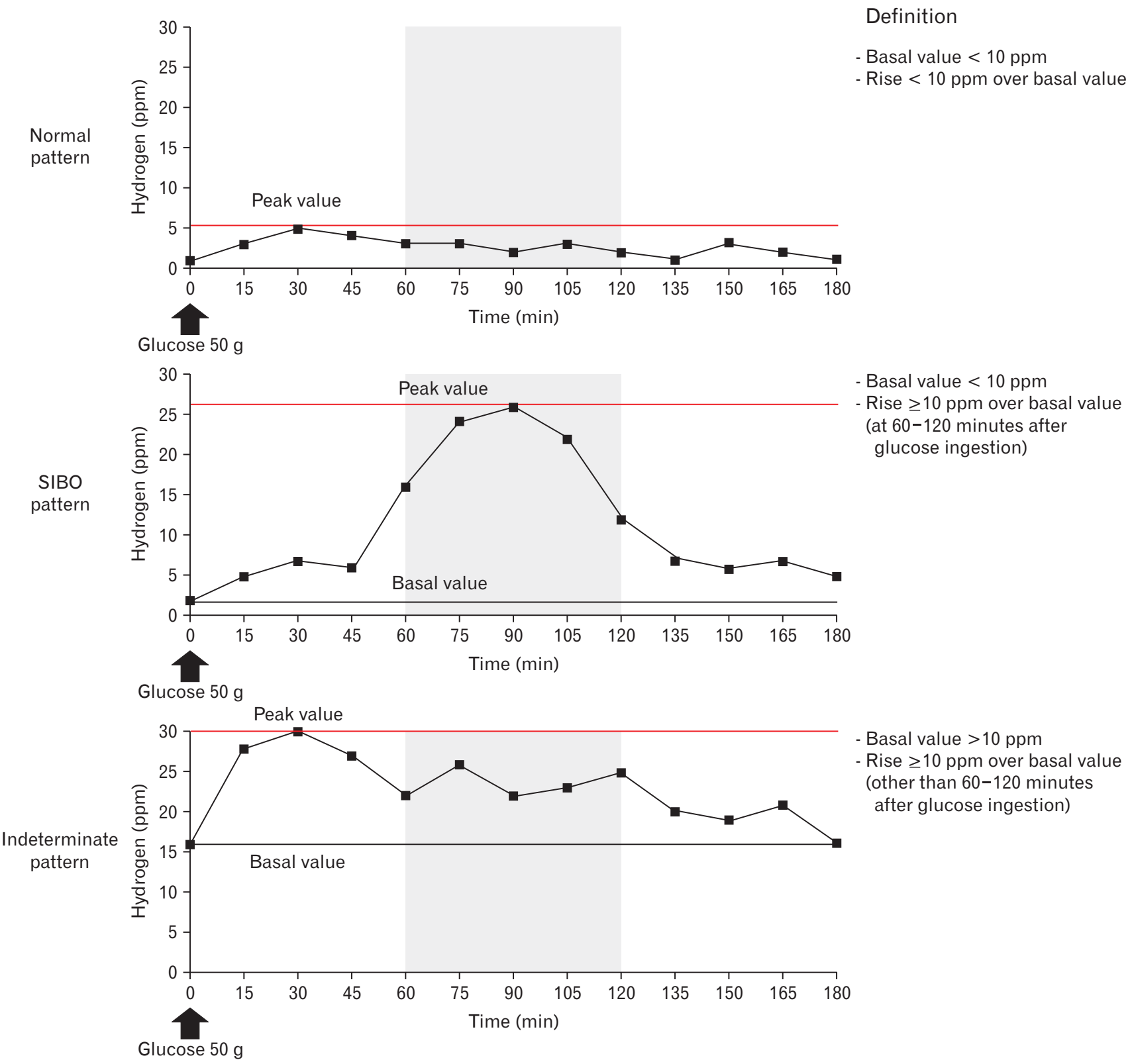

Figure 1. Patterns shown by glucose hydrogen breath test findings obtained by administering $50 \mathrm{~g}$ of glucose to the subjects. The results were classified as normal, small intestinal bacterial overgrowth (SIBO), and indeterminate patterns. SIBO was diagnosed when the basal value was $<10$ ppm and the subtracted peak value above the basal level was $\geq 10$ ppm at 60-120 minutes after glucose loading.

tion, symptoms were assessed before and after the end of treatment using the Izumo scale.

\section{Ethical Approval}

The protocol of this study was evaluated and approved by the ethical committee of Shimane University School of Medicine, and informed consent was obtained from all enrolled patients prior to participation. This study was registered with the University Hospi- tal Medical Information Network (UMIN) clinical trials registry (UMIN 000013545).

\section{Results}

\section{Clinical Characteristics of Enrolled Patients}

Thirty-eight patients (18 men, 20 women; mean age 56.0 
Table 2. Clinical Characteristics of Enrolled Subjects

\begin{tabular}{|c|c|}
\hline Number of cases & 38 \\
\hline Gender (male:female) & $18: 20$ \\
\hline Age (mean $\pm \mathrm{SD}$ [range]), yr & $56.0 \pm 17.1(19-81)$ \\
\hline Baseline Izumo scale score (median [range]) & $22(6-62)$ \\
\hline \multicolumn{2}{|l|}{ Initial diagnosis } \\
\hline FD & $28(73.7 \%)$ \\
\hline PDS & 24 \\
\hline EPS & 4 \\
\hline IBS & $27(71.1 \%)$ \\
\hline IBS-C & 10 \\
\hline IBS-D & 11 \\
\hline IBS-M & 6 \\
\hline (overlapped FD and IBS) & $17(44.7 \%)$ \\
\hline \multicolumn{2}{|l|}{ Most bothersome symptom (duplicates counted) } \\
\hline Bloating & $27(71.1 \%)$ \\
\hline Abnormal bowel movement & $19(50.0 \%)$ \\
\hline Abdominal pain & $11(28.9 \%)$ \\
\hline Rugitus & $5(13.2 \%)$ \\
\hline Others & $4(10.5 \%)$ \\
\hline \multicolumn{2}{|l|}{ Initial treatment (duplicates counted) } \\
\hline Prokinetics & $17(44.7 \%)$ \\
\hline Antidepressant & $10(26.3 \%)$ \\
\hline Laxative & $9(23.7 \%)$ \\
\hline Proton pump inhibitor & $7(18.4 \%)$ \\
\hline $\mathrm{H}_{2}$ receptor antagonist & $4(10.5 \%)$ \\
\hline Others $^{a}$ & $8(21.1 \%)$ \\
\hline
\end{tabular}

${ }^{a}$ Others included herbal medicine (Rikkunshito and Daikenchuto) and antidiarrheal drug (loperamide).

FD, functional dyspepsia; PDS, post prandial distress syndrome; EPS, epigastric pain syndrome; IBS, irritable bowel syndrome; IBS-C, IBS with constipation; IBS-D, IBS with diarrhea; IBS-M, mixed IBS.

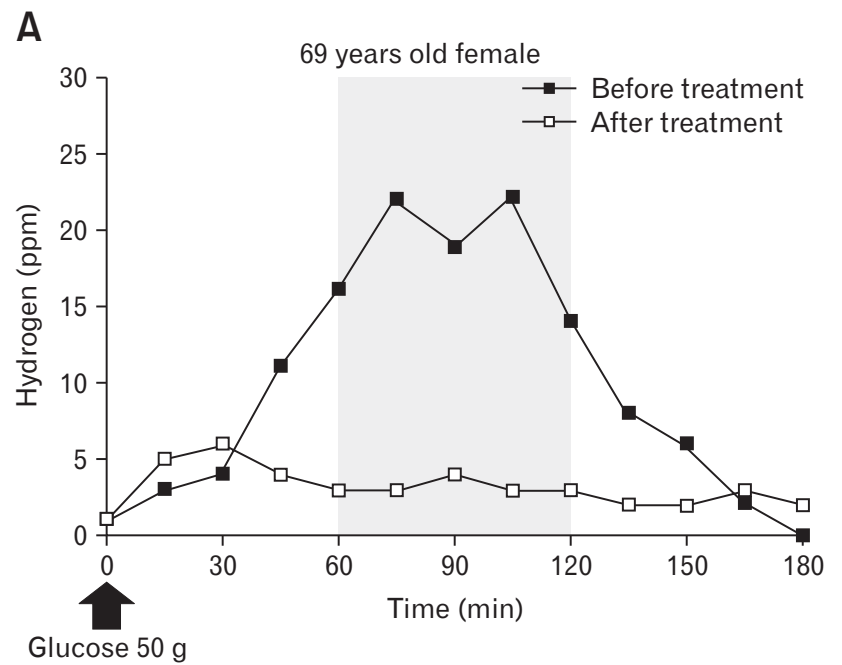

years, range 19-81 years) with refractory FGID were enrolled in this study (Table 2). Of those 38, 11 were diagnosed with FD, 10 with IBS, and 17 with overlapping FD and IBS at enrollment. Each subtype and clinical characteristics were shown in Table 2. Approximately $70 \%$ of the patients $(n=27)$ complained of bloating or abdominal fullness, while 19 (50.0\%) noted abnormal bowel movements and 10 (26.3\%) abdominal pain. Initial treatment for the enrolled patients included prokinetics $(n=17,44.7 \%)$, antidepressants $(\mathrm{n}=10,26.3 \%)$, laxatives $(\mathrm{n}=9,23.7 \%)$, PPIs $(\mathrm{n}=7$, $18.4 \%)$, histamine-2 blockers $(\mathrm{n}=4,10.5 \%)$, and others $(\mathrm{n}=8$, $21.1 \%)$.

\section{Frequency of Small Intestinal Bacterial Overgrowth}

We firstly conducted GHBT in healthy volunteer. All the healthy adults showed a GHBT normal pattern (data not shown). Thirty-four $(89.5 \%)$ of the 38 enrolled patients showed a GHBT normal pattern, while 2, including 1 with FD and 1 with IBS, were classified as the indeterminate pattern, both of whom showed a fasting breath hydrogen level $>20 \mathrm{ppm}$. The exact cause of high basal hydrogen value in these cases remains unclear. Only 2 (5.3\%) patients were finally diagnosed with SIBO, and their GHBT patterns and clinical features are shown in Figure 2 and Table 3, respectively. Both of the SIBO patients were female, and negative for Helicobacter pylori infection. Case A was initially diagnosed overlapped with PDS and IBS-M, while Case B was diagnosed with PDS at enrollment. PPI, prokinetic, and antidepressant administrations were prescribed for case A at enrollment, while prokinetic was pre-

\section{B}

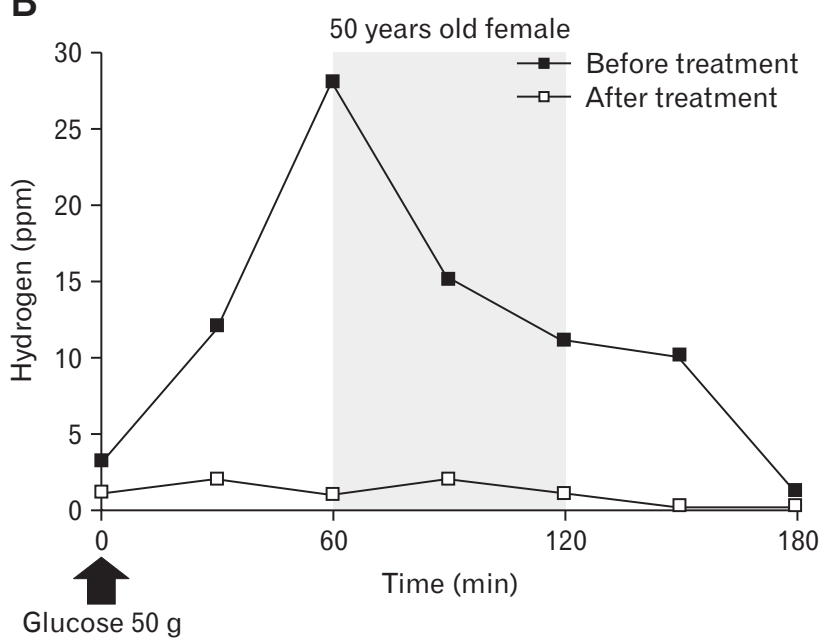

Figure 2. Patterns shown by glucose hydrogen breath test findings in 2 patients with small intestinal bacterial overgrowth before and after levofloxacin administration. (A) Case A. (B) Case B. 
Table 3. Clinical Characteristics of 2 Patients with Small Intestinal Bacterial Overgrowth

\begin{tabular}{|c|c|c|}
\hline & Case A & Case B \\
\hline Gender & Female & Female \\
\hline Age (yr) & 69 & 50 \\
\hline Most bothersome symptom & Bloating and belching & Rugitus \\
\hline Initial diagnosis & Overlapped FD (PDS) and IBS-M & FD (PDS) \\
\hline Helicobacter pylori infection & Negative & Negative \\
\hline \multirow[t]{3}{*}{ Initial treatment } & Esomeprazole & Mosapride \\
\hline & Mosapride & \\
\hline & Paroxetine & \\
\hline Treatment & Levofloxacin & Levofloxacin \\
\hline \multicolumn{3}{|l|}{ Izumo scale score $\mathrm{a}^{\mathrm{a}}$} \\
\hline Before treatment & $32(4,0,12,8,8)$ & $17(4,3,4,4,2)$ \\
\hline After treatment & $26(5,0,4,8,9)$ & $8(4,2,0,2,0)$ \\
\hline \multicolumn{3}{|l|}{ GHBT before treatment } \\
\hline Basal & $1 \mathrm{ppm}$ & $3 \mathrm{ppm}$ \\
\hline Peak (time) & $22 \mathrm{ppm}$ (at 75 and $105 \mathrm{~min}$ ) & $28 \mathrm{ppm}$ (at $60 \mathrm{~min}$ ) \\
\hline \multicolumn{3}{|l|}{ GHBT after treatment } \\
\hline Basal & $1 \mathrm{ppm}$ & $1 \mathrm{ppm}$ \\
\hline Peak (time) & $6 \mathrm{ppm}$ (at $30 \mathrm{~min}$ ) & $2 \mathrm{ppm}$ (at 30 and $90 \mathrm{~min}$ ) \\
\hline
\end{tabular}

${ }^{a}$ Numbers in parentheses indicate scores in each domain; reflux, pain, fullness, constipation, and diarrhea, respectively.

FD, functional dyspepsia; PDS, postprandial distress syndrome; IBS-M, mixed irritable bowel syndrome; GHBT, glucose hydrogen breath test.

scribed for case B. Case A complained of bloating and belching, while case $\mathrm{B}$ complained of rugitus.

\section{Assessment of Treatment}

The 2 patients with SIBO were treated with levofloxacin at $500 \mathrm{mg}$ for 7 days and no adverse events were reported. GHBT was performed again after the end of treatment and both patients showed a normal pattern (Fig. 2). In addition, their symptoms were also clearly improved. Scores on fullness domain of the Izumo scale were decreased after treatment in both cases (12 to 4 in case $A$ and 4 to 0 in case $B$ ), with little or no change of the other symptom domains (Table 3).

\section{Discussion}

In the present study, we evaluated the frequency of SIBO in Japanese patients with refractory FGID. Good control of FGID by medical treatment may be less possible for SIBO patients, thus we focused on those with refractory FGID. Among the 38 prospectively enrolled patients with refractory FGID, only 2 (5.3\%) were diagnosed with SIBO as assessed by GHBT, suggesting that the frequency may be low in Japan.

SIBO is a very heterogeneous syndrome characterized by an increased number and/or abnormal types of bacteria in the small intestine. Wide variations in prevalence rates of SIBO have been reported in patients with FGID. As for IBS, to date, over 20 studies of the frequency of SIBO in affected patients have reported prevalence ranging from $2 \%$ to $84 \% .^{34,35}$ Most of those studies suggested that patients with IBS have a greater probability of SIBO. ${ }^{36}$ In contrast, few studies have reported the prevalence of SIBO in patients with FD. In a study conducted in Brazil, Costa et $\mathrm{al}^{16}$ noted that $56.5 \%$ (13/23) of FD patients were diagnosed with SIBO by LHBT. In contrast, our study conducted in Japan found only 2 (7.1\%) of 28 FD patients, including 10 cases overlapped with IBS, diagnosed with SIBO. Both FD were subtyped as PDS. Lower prevalence of SIBO in FGID in a Japanese population remains unclear. Clustering small bowel microbiome may be different among geographic areas. As for dietary habits, complex carbohydrates, which are major source of calories for most of the world's population, varies hydrogen excretion after ingestion. Breath hydrogen concentration is significantly elevated after ingestion of carbohydrate including white wheat, beans, oats, potatoes, and corn, while, white rice, which is staple food of the Japanese, is the only complex carbohydrate source that was nearly completely absorbed. ${ }^{37}$ Taken together, factors such as dietary habits, geography, ethnicity, and lifestyle can affect enteric bacteria colonization and influence the prevalence of SIBO. Variance of these factors may explain the different prevalence rates of SIBO in patients with FGID throughout 
the world.

Such variations may also be explained by results of different diagnostic tests to establish SIBO and differing diagnostic criteria for FGID. Traditionally, findings of a jejunal aspirate culture, performed with fluid sampled from the small intestine, has been considered to be the gold standard for diagnosis of SIBO. However, various problems, including invasiveness, possible sample contamination, low accessibility, and inability to diagnose distal small bowel bacterial overgrowth, have been pointed out. ${ }^{14,38}$ The diagnostic yield of breath tests for SIBO in previous studies has also varied, because of differences in the dose and type of substrate used, threshold for defining a positive breath test, and analysis of breath samples for hydrogen alone or a combination of hydrogen and methane. ${ }^{15,39}$ However, a breath test is likely to be conducted as the first choice for diagnosis of SIBO in clinical practice because of low cost, high safety, and availability. GHBT and LHBT are the most common breath tests, though both have distinct limitations. ${ }^{14,21,31}$ In this study, we conducted GHBT with $50 \mathrm{~g}$ of glucose and defined a rise of $\geq 10 \mathrm{ppm}$ over baseline as diagnostic of SIBO, based on the data in healthy volunteer. However, our 2 cases of SIBO had a rise of $>20 \mathrm{ppm}$. Therefore, our SIBO patients may have be diagnosed with criteria different from those used in previous clinical trials using peak value above the basal $>20$ ppm. ${ }^{15,40-42}$ Nonetheless, the advent of techniques using nucleic acid-based strategies and metagenomics to define the gut ecosystem may change the definition used for diagnosis of SIBO in the future.

Another methodological issue in hydrogen breath test is a general lack of standardization for withdrawal period of affecting drugs before testing. The use of antibiotics or probiotics definitely modifies the composition of intestinal microflora and may therefore be a cause of interference with the test results. Likewise, laxatives and electrolyte solutions for colonic cleaning may interfere with the stability of colonic microflora. Therefore, a test delay is recommended in such cases. ${ }^{13,15}$ Prokinetics are also recommended to stop administration for 4 weeks before testing. ${ }^{15}$ However, it remains to be elucidated whether prokinetics are responsible for modification of intestinal microflora. In this study, the patients who had taken antibiotics or probiotics within 4 weeks preceding the test were excluded. No one used laxatives for colonic purging before colonoscopy within 4 weeks preceding the test, while, laxatives such as senna to help daily bowel movement were used in 9 cases. In these cases, drugs were withdrawn 24 hours before testing. Nevertheless, the effect of these drugs on the modification of intestinal microflora should be fully clarified in the future. The profound gastric acid suppression induced by long-term PPI treatment may alter intes- tinal microflora, ${ }^{43,44}$ thus PPI administration has been suggested to be a risk factor for development of SIBO, though that relationship remains undetermined. Some studies have found no correlation between PPI use and SIBO as measured by indirect breath tests, whereas others that used small bowel aspirates showed a higher association of SIBO with PPI administration as compared to the least potent gastric antisecretory therapy. ${ }^{45-48}$ In the present cohort, PPIs were administered for FD symptoms in 7 (18.4\%) of the enrolled patients, 1 of whom was diagnosed with SIBO. However, the symptoms in that latter patient did not improve after stopping PPI administration, suggesting that the symptoms were not caused by the PPI.

Once a diagnosis of SIBO is established, antibiotic therapy is aimed at reducing or eliminating bacterial overgrowth, accompanied by resolution of symptoms. However, there is no consensus regarding the antibiotic of choice for SIBO. Recent studies have shown that rifaximin, a semisynthetic antibacterial derivative of rifampicin with virtually no systemic absorption and a favorable sideeffect profile, is mainly used for treatment. ${ }^{33}$ However, rifaximin is not available in Japan, thus we prescribed levofloxacin for 7 days to our SIBO patients and their symptoms were significantly improved along with a decreased level of hydrogen after that administration. Recurrence of symptoms after the treatment course is an important issue to manage $\mathrm{SIBO}$ patients. Lauritano et $\mathrm{al}^{49}$ used rifaximin at $400 \mathrm{mg} 3$ times daily for 10 days to treat 80 consecutive patients with IBS and SIBO, then the presence of SIBO was examined at 3 , 6, and 9 months after the end of treatment. Recurrence was documented in $12.6 \%$ of those patients at 3 months, $27.5 \%$ at 6 months, and $43.7 \%$ at 9 months. ${ }^{49}$ These results suggest a need for further treatment in many affected patients. One of our 2 SIBO patients had recurrent symptoms with high breath hydrogen gas concentration shown by GHBT after 12 months of treatment. It is considered difficult to diagnose SIBO by a symptom questionnaire alone and Jacobs et al ${ }^{46}$ reported that symptom profiles were poor predictors of SIBO. Therefore, the noninvasive GHBT may be useful for surveillance of SIBO during a long term follow-up course.

Although methane production is considered to be associated with SIBO, we did not measure methane concentration in breath samples in the present study, thus the frequency of SIBO may have been underestimated. Indeed, $8 \%$ to $27 \%$ of human subjects did not have detectable hydrogen production from their gastrointestinal microbiota, but rather produced methane. ${ }^{15,50,51}$ In contrast, Rana et $\mathrm{al}^{31}$ reported that the presence of methane did not have a significant impact on their investigation of SIBO in patients with IBS. To complement the low sensitivity of the method, an alterna- 
tive approach to measure both hydrogen and methane at expiration is suggested. Other limitations of the present study include the low number of enrolled patients. Also, $H$. pylori infection status was not confirmed in some cases, while the cases with SIBO was confirmed as negative for $H$. pylori infection. Furthermore, our results were obtained from a selected population treated at a tertiary care center, thus they should be interpreted with caution and may not be generalizable to the population at large.

In conclusion, based on our findings, SIBO is rare in Japanese patients with FD and IBS. The 2 patients with SIBO showed clearly improved abdominal symptoms along with decreased hydrogen expiration after administration of antibiotics. Although seemingly rare, it is important to consider SIBO as a differential diagnosis in patients with refractory $\mathrm{FD}$ and IBS.

Acknowledgements: The authors thank Seiji Odagawa (Oki Hospital) for the important contributions to this study.

\section{Financial support: None.}

\section{Conflicts of interest: None.}

Author contributions: Shino Shimura, Norihisa Ishimura, Shunji Ishihara, and Yoshikazu Kinoshita contributed to the conception and design of the study, collection, assembly and interpretation of the data, and the drafting of the manuscript; Hironobu Mikami, Eiko Okimoto, Goichi Uno, and Yuji Tamagawa contributed to the conception and design of the study, and the analysis and interpretation of the data; and Masahito Aimi, Shuichi Sato, and Naoki Oshima contributed to the statistical analysis plan for the study, and the analysis and interpretation of the data. All authors read and approved the final manuscript.

\section{References}

1. Tack J, Talley NJ, Camilleri M, et al. Functional gastroduodenal disorders. Gastroenterology 2006;130:1466-1479.

2. Okimoto E, Ishimura N, Morito Y, et al. Prevalence of gastroesophageal reflux disease in children, adults, and elderly in the same community. J Gastroenterol Hepatol 2015;30:1140-1146.

3. Oshima T, Miwa H. Epidemiology of functional gastrointestinal disorders in Japan and in the world. J Neurogastroenterol Motil 2015;21:320329.

4. Lovell RM, Ford AC. Global prevalence of and risk factors for irritable bowel syndrome: a meta-analysis. Clin Gastroenterol Hepatol 2012;10: 712-721, e4.

5. Miwa H. Why dyspepsia can occur without organic disease: pathogenesis and management of functional dyspepsia. J Gastroenterol 2012;47:862871.

6. Oshima T, Fukui H, Watari J, Miwa H. Childhood abuse history is associated with the development of dyspepsia: a population-based survey in Japan. J Gastroenterol 2015;50:744-750.

7. Shulman RJ, Jarrett ME, Cain KC, Broussard EK, Heitkemper MM. Associations among gut permeability, inflammatory markers, and symptoms in patients with irritable bowel syndrome. J Gastroenterol 2014;49: 1467-1476.

8. Walker MM, Aggarwal KR, Shim LS, et al. Duodenal eosinophilia and early satiety in functional dyspepsia: confirmation of a positive association in an Australian cohort. J Gastroenterol Hepatol 2014;29:474-479.

9. Seo AY, Kim N, Oh DH. Abdominal bloating: pathophysiology and treatment. J Neurogastroenterol Motil 2013;19:433-453.

10. Simrén M, Barbara G, Flint HJ, et al. Intestinal microbiota in functional bowel disorders: a Rome foundation report. Gut 2013;62:159-176.

11. Tana C, Umesaki Y, Imaoka A, Handa T, Kanazawa M, Fukudo S. Altered profiles of intestinal microbiota and organic acids may be the origin of symptoms in irritable bowel syndrome. Neurogastroenterol Motil 2010;22:512-519, e114-e115.

12. Grace E, Shaw C, Whelan K, Andreyev HJ. Review article: small intestinal bacterial overgrowth--prevalence, clinical features, current and developing diagnostic tests, and treatment. Aliment Pharmacol Ther 2013;38:674-688.

13. Saad RJ, Chey WD. Breath testing for small intestinal bacterial overgrowth: maximizing test accuracy. Clin Gastroenterol Hepatol 2014;12: 1964-1972.

14. Gabrielli M, D’Angelo G, Di Rienzo T, Scarpellini E, Ojetti V. Diagnosis of small intestinal bacterial overgrowth in the clinical practice. Eur Rev Med Pharmacol Sci 2013;17(suppl 2):30-35.

15. Gasbarrini A, Corazza GR, Gasbarrini G, et al. Methodology and indications of H2-breath testing in gastrointestinal diseases: the Rome Consensus Conference. Aliment Pharmacol Ther 2009;29(suppl 1):1-49.

16. Costa MB, Azeredo Jr IL, Marciano RD, Caldeira LM, Bafutto M. Evaluation of small intestine bacterial overgrowth in patients with functional dyspepsia through H2 breath test. Arq Gastroenterol 2012;49:279283.

17. Parodi A, Dulbecco P, Savarino E, et al. Positive glucose breath testing is more prevalent in patients with IBS-like symptoms compared with controls of similar age and gender distribution. J Clin Gastroenterol 2009;43:962-966.

18. Shah ED, Basseri RJ, Chong K, Pimentel M. Abnormal breath testing in IBS: a meta-analysis. Dig Dis Sci 2010;55:2441-2449.

19. Ford AC, Spiegel BM, Talley NJ, Moayyedi P. Small intestinal bacterial overgrowth in irritable bowel syndrome: systematic review and metaanalysis. Clin Gastroenterol Hepatol 2009;7:1279-1286.

20. Schmulson M, Bielsa MV, Carmona-Sánchez R, et al. Microbiota, gastrointestinal infections, low-grade inflammation, and antibiotic therapy in irritable bowel syndrome: an evidence-based review. Rev Gastroenterol Mex 2014;79:96-134.

21. Wang Y, Xiong L, Gong X, Li W, Zhang X, Chen M. Small intestinal bacterial overgrowth as an uncommon cause of false positive lactose hy- 
drogen breath test among patients with diarrhea-predominant irritable bowel syndrome in Asia. J Gastroenterol Hepatol 2015;30:995-1000.

22. Longstreth GF, Thompson WG, Chey WD, Houghton LA, Mearin F, Spiller RC. Functional bowel disorders. Gastroenterology 2006;130:1480-1491.

23. Furuta K, Ishihara S, Sato S, et al. [Verification of responsiveness in the Izumo Scale for patients with gastrointestinal symptoms.] Ther Res 2009;30:1651-1658. [Japanese]

24. Furuta K, Ishihara S, Sato S, et al. [Development and verification of the Izumo Scale, new questionnaire for quality of life assessment of patients with gastrointestinal symptoms.] Nihon Shokakibyo Gakkai Zasshi 2009;106:1478-1487. [Japanese]

25. Kakuta E, Yamashita N, Katsube T, et al. Abdominal symptom-related QOL in individuals visiting an outpatient clinic and those attending an annual health check. Intern Med 2011;50:1517-1522.

26. Kinoshita Y, Chiba T, FUTURE study group. Therapeutic effects of famotidine on chronic symptomatic gastritis: subgroup analysis from FUTURE study. J Gastroenterol 2012;47:377-386.

27. Lewis SJ, Heaton KW. Stool form scale as a useful guide to intestinal transit time. Scand J Gastroenterol 1997;32:920-924.

28. Babu J, Kumar S, Babu P, Prasad JH, Ghoshal UC. Frequency of lactose malabsorption among healthy southern and northern Indian populations by genetic analysis and lactose hydrogen breath and tolerance tests. Am J Clin Nutr 2010;91:140-146.

29. Kumar S, Misra A, Ghoshal UC. Patients with irritable bowel syndrome exhale more hydrogen than healthy subjects in fasting state. J Neurogastroenterol Motil 2010;16:299-305.

30. Murray K, Wilkinson-Smith V, Hoad C, et al. Differential effects of FODMAPs (fermentable oligo-, di-, mono-saccharides and polyols) on small and large intestinal contents in healthy subjects shown by MRI. Am J Gastroenterol 2014;109:110-119.

31. Rana SV, Sharma S, Kaur J, Sinha SK, Singh K. Comparison of lactulose and glucose breath test for diagnosis of small intestinal bacterial overgrowth in patients with irritable bowel syndrome. Digestion 2012;85:243247.

32. Pimentel M. Review of rifaximin as treatment for SIBO and IBS. Expert Opin Investig Drugs 2009;18:349-358.

33. Shah SC, Day LW, Somsouk M, Sewell JL. Meta-analysis: antibiotic therapy for small intestinal bacterial overgrowth. Aliment Pharmacol Ther 2013;38:925-934.

34. Ghoshal UC, Kumar S, Mehrotra M, Lakshmi C, Misra A. Frequency of small intestinal bacterial overgrowth in patients with irritable bowel syndrome and chronic non-specific diarrhea. J Neurogastroenterol Motil 2010;16:40-46.

35. Pimentel M, Chow EJ, Lin HC. Eradication of small intestinal bacterial overgrowth reduces symptoms of irritable bowel syndrome. Am J Gas- troenterol 2000;95:3503-3506

36. Ghoshal UC, Srivastava D. Irritable bowel syndrome and small intestinal bacterial overgrowth: meaningful association or unnecessary hype. World J Gastroenterol 2014;20:2482-2491.

37. Levitt MD, Hirsh P, Fetzer CA, Sheahan M, Levine AS. H2 excretion after ingestion of complex carbohydrates. Gastroenterology 1987;92:383389.

38. Pyleris E, Giamarellos-Bourboulis EJ, Tzivras D, Koussoulas V, Barbatzas $\mathrm{C}$, Pimentel $\mathrm{M}$. The prevalence of overgrowth by aerobic bacteria in the small intestine by small bowel culture: relationship with irritable bowel syndrome. Dig Dis Sci 2012;57:1321-1329.

39. Shin W. Medical applications of breath hydrogen measurements. Anal Bioanal Chem 2014;406:3931-3939.

40. Ghoshal UC. How to interpret hydrogen breath tests. J Neurogastroenterol Motil 2011;17:312-317.

41. Goebel-Stengel M, Stengel A, Schmidtmann M, Voort Iv, Kobelt P, Monnikes H. Unclear abdominal discomfort: pivotal role of carbohydrate malabsorption. J Neurogastroenterol Motil 2014;20:228-235.

42. Simrén M, Stotzer PO. Use and abuse of hydrogen breath tests. Gut 2006;55:297-303.

43. Feldman M. Gastric acid secretion: still relevant? Am J Gastroenterol 2013;108:347-352.

44. Sachs G, Shin JM, Munson K, Scott DR. Gastric acid-dependent diseases: a twentieth-century revolution. Dig Dis Sci 2014;59:1358-1369.

45. Compare D, Pica L, Rocco A, et al. Effects of long-term PPI treatment on producing bowel symptoms and SIBO. Eur J Clin Invest 2011;41: 380-386.

46. Jacobs C, Coss Adame E, Attaluri A, Valestin J, Rao SS. Dysmotility and proton pump inhibitor use are independent risk factors for small intestinal bacterial and/or fungal overgrowth. Aliment Pharmacol Ther 2013;37:1103-1111.

47. Lo WK, Chan WW. Proton pump inhibitor use and the risk of small intestinal bacterial overgrowth: a meta-analysis. Clin Gastroenterol Hepatol 2013;11:483-490.

48. Ratuapli SK, Ellington TG, O’Neill MT, et al. Proton pump inhibitor therapy use does not predispose to small intestinal bacterial overgrowth. Am J Gastroenterol 2012;107:730-735.

49. Lauritano EC, Gabrielli M, Scarpellini E, et al. Small intestinal bacterial overgrowth recurrence after antibiotic therapy. Am J Gastroenterol 2008; 103:2031-2035.

50. Attaluri A, Jackson M, Valestin J, Rao SS. Methanogenic flora is associated with altered colonic transit but not stool characteristics in constipation without IBS. Am J Gastroenterol 2010;105:1407-1411.

51. Triantafyllou K, Chang C, Pimentel M. Methanogens, methane and gastrointestinal motility. J Neurogastroenterol Motil 2014;20:31-40. 